\title{
Dead-beat and reaching law based sliding mode control laws for perishable inventories with transportation losses
}

\author{
ANDRZEJ BARTOSZEWICZ and MICHAŁ MACIEJEWSKI
}

\begin{abstract}
This paper describes discrete sliding mode (SM) supply management strategies for inventory systems with perishable goods and transportation losses. In the considered systems, the stock used to satisfy the unknown, bounded and time-varying demand is replenished with some delay from a distant supply source. The on-hand stock deterioration during lead-time delay, as well as commodity losses in supply process are explicitly taken into account. Two supply management strategies are proposed. The first one ensures fast reaction to the imposed demand variations, but may result in excessive control signal magnitude at the beginning of the inventory management process. Therefore, in order to conform to supplier limitations we also develop an alternative control strategy based on the concept of the reaching law. That strategy helps reduce the initial supply rate and satisfy the supplier limitations. A number of desirable properties of both proposed strategies are formulated and formally proved. These properties include full customer demand satisfaction and elimination of the risk of exceeding the warehouse capacity.
\end{abstract}

Key words: sliding mode control, perishable inventories, inventory control

\section{Introduction}

The control theoretic approach to the management of logistic processes, and in particular to the problem of supply chain management has recently become an important research subject. A good overview of the techniques used in the field and the obtained results can be found in $[5,12,20,23]$. The first application of the control theory methods to the management of logistic processes was reported in the early 1950s when Simon [24] applied servomechanism control algorithm to find an efficient strategy of goods replenishment in continuous time, single product inventory control systems. A few years later the discrete time servomechanism control algorithm for the purpose of efficient goods replenishment has been proposed [26]. Since that time numerous solutions have

The Authors are with Institute of Automatic Control, Technical University of Łódź, 18/22 Stefanowskiego St., 90-924 Łódź, Poland. E-mails: andrzej.bartoszewicz@p.lodz.pl, maciejewskimichal@ o2.pl

This work has been performed in the framework of a project "Optimal sliding mode control of time delay systems" financed by the National Science Centre of Poland - decision number DEC 2011/01/B/ST7/02582.

Received 03.07.2012. 
been presented, and therefore, further in this chapter we are able to mention only a few, arbitrarily selected examples of solutions proposed over the last decades. In [8] and [9] autoregressive moving average (ARMA) system structure has been applied in order to model uncertain demand. Then in [1] and [21] model predictive control of supply chain has been proposed and in [6] a robust controller for the continuous-time system with uncertain processing time and delay has been designed by minimizing $H_{\infty}$-norm. However, practical implementation of the strategy described in [6] requires application of numerical methods in order to obtain the control law parameters, which limits its analytical tractability.

Furthermore, in [15] lead-time delay is explicitly taken into account and represented by additional state variables in the state space description. This approach results in the optimal controller designed by minimization of quadratic performance index. A similar approach is applied in [17] where an LQ optimal sliding mode controller is designed. In [16] LQ optimal sliding mode is compared with nonlinear controller based on the concept of reaching law. However, papers $[15,16,17]$ are concerned with conventional, non-deteriorating inventories only. An extension of the results presented in [15] to the case of perishable inventories is given in [14]. An LQ optimal sliding mode controller for supply chains with deteriorating stock is proposed in [18] and the idea of reaching law is applied to the design of sliding mode controller for systems with deteriorating stock in [19]. However, none of the papers [14-19] takes into account transportation losses (or in other words requested goods decay during the order procurement time). Therefore, in this paper we consider perishable inventories and we explicitly account for the ordered goods losses during the lead time.

Similarly as in [14], [18] and [19], in this paper we also consider a periodic-review inventory system with perishable goods. However, on the contrary to the previously published results we consider not only losses which take place when the commodity is stored in the warehouse, but also those which happen during the supply process, i.e. the losses on the way from the supplier to the warehouse. We propose a discrete time representation of the supply chain dynamics and we apply discrete time sliding mode methodology $[2,3,4,7,10,11,22,25]$ to design the controller for the considered system. The controller design objective is on one hand to fully satisfy the imposed demand, and on the other, to minimize at the same time the on-hand inventory volume. This reflects the need of simultaneous minimization of the lost sales costs and inventory holding costs. Since the demand may vary quite rapidly, we determine the sliding hyperplane so that the proposed discrete time sliding mode controller ensures the dead-beat system performance. Hence the closed-loop system is stabilized and its error converges to zero in the shortest possible time. This approach results in good dynamics of the closed loop system and its fast reaction to the unpredictable variations of demand. Moreover, the sliding mode controller proposed further in this paper leads to chattering free system operation. The controller is determined analytically in a closed form, which allows us to state and formally prove important properties of the proposed inventory policy. First, we prove that the designed management policy always generates strictly positive and upper bounded order quantities, which is an important issue from the practical point of view. Next, we 
define the warehouse capacity which provides enough space for all incoming shipments, and finally we show that all the imposed demand is fully satisfied, which guarantees $100 \%$ service level. As the proposed strategy generates high initial order quantity, which is an undesirable effect, further in the paper we employ the concept of reaching law in the form described in [11]. By this means we obtain a modified control structure which maintains good system dynamics offered by the dead-beat scheme, and simultaneously guarantees that supplier capacity limitations are satisfied. We describe the proposed procedure of finding the reaching law parameters, and we state properties of the modified controller in two theorems. The results reported in this paper may be seen as an extension of our previous work reported in [19] to the case of more general inventory management systems, i.e. the supply chains with transportation losses explicitly taken into account.

\section{Problem statement}

In this paper we consider a production-inventory system where distribution centre, replenished from a single supply source, provides products for customers or another production stage. The flow of goods and information in the considered system (with transportation losses and the on-hand stock deterioration) is illustrated in Fig. 1.

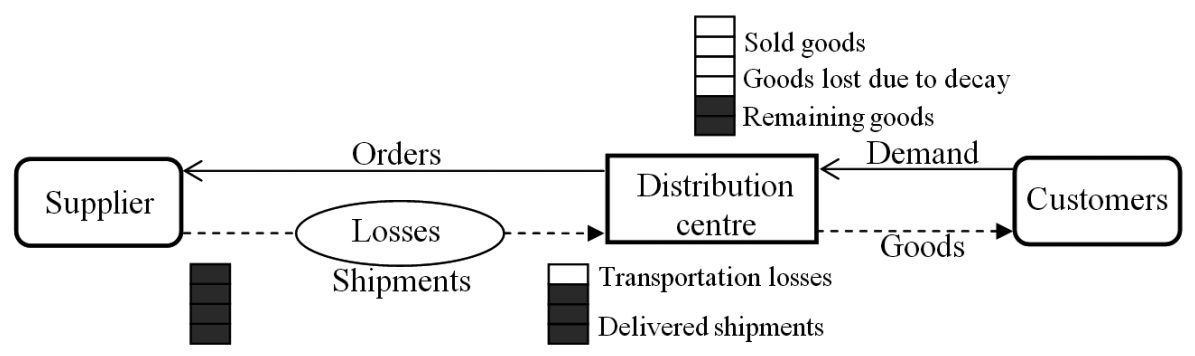

Figure 1. Flow of goods and information in the considered system.

The analyzed inventory system is subject to an a priori unknown, bounded, timevarying demand. The main objective of this work is to design a stable supply policy, which will maximize demand satisfaction from the resources available at the distribution centre. The design procedure proposed in the paper not only explicitly takes into account the delay (lead-time) between placing of an order at the supplier and goods arrival at the distribution centre, but it also directly accounts for the on-hand stock deterioration and commodity losses in the supply process, i.e. the losses which take place during the leadtime. The model of the analyzed inventory system is presented in Fig. 2.

The stock replenishment orders $u(k T)$ are placed at regular time instants $k T$, where $T$ is the review period of the considered process and $k=0,1,2, \ldots$. The particular value of each order is calculated on the basis of the current stock level $y(k T)$, the stock reference 
level $y_{r e f}$ and the order history. The orders arrive at the distribution centre after leadtime delay $L_{p}$, which is a multiple of the review period. Thus, $L_{p}=n_{p} T$, where $n_{p}$ is a positive integer.

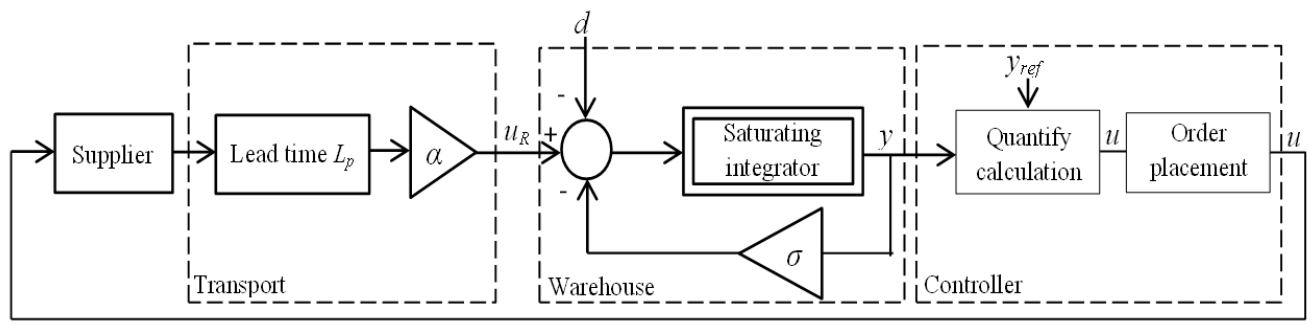

Figure 2. System model.

The imposed demand (the amount of goods requested from inventory in period $k$ ) is modeled as an unknown, bounded function of time $0 \leqslant d(k T) \leqslant d_{\max }$. It is worth to notice, that this definition of the demand is quite general and makes the presented approach fairly universal. According to this definition the following two situations may occur:

- If there is a sufficient amount of goods in the warehouse, then the imposed demand is fully satisfied.

- If the imposed demand is greater than the amount of goods available at the on-hand stock and in arriving shipments, then only some part of the demand is satisfied. Hence, additional demand is lost, as we assume that the sales are not backordered.

Let $h(k T)$ denote the amount of goods sold to customers or sent to retailers in the distribution network at time instant $k T$. Then

$$
0 \leqslant h(k T) \leqslant d(k T) \leqslant d_{\max } .
$$

Stock balance equation for the considered system with perishable inventory has the following form

$$
y[(k+1) T]=\rho y(k T)+u_{R}(k T)-h(k T)
$$

where $u_{R}(k T)$ is the order received at time $k T$. The fraction of perishable stock which remains in the warehouse after each review period is represented by $\rho=1-\sigma$. We assume that incoming shipments also deteriorate during transportation process. Consequently, the fraction of ordered goods which arrive at the warehouse is represented by $\alpha$, where $0<\alpha \leqslant 1$. Thus,

$$
u_{R}(k T)=\alpha u\left[\left(k-n_{p}\right) T\right] .
$$

Furthermore, we assume that the warehouse is initially empty, i.e. $y(k T)=0$ for $k<0$, and the first order is placed at the time instant $k T=0$. Due to the lead-time delay, 
the first order arrives at the warehouse at the time instant $n_{p}$, and $y(k T)=0$ for any $k \leqslant n_{p}$. Taking into account initial conditions and (3), the stock level for any $k>0$ may be expressed as

$$
\begin{aligned}
y(k T) & =\sum_{j=0}^{k-1} \rho^{k-1-j} u_{R}(j T)-\sum_{j=0}^{k-1} \rho^{k-1-j} h(j T)= \\
& =\sum_{j=0}^{k-1} \alpha \rho^{k-1-j} u\left[\left(j-n_{p}\right) T\right]-\sum_{j=0}^{k-1} \rho^{k-1-j} h(j T)= \\
& =\alpha \sum_{j=0}^{k-n_{p}-1} \rho^{k-n_{p}-1-j} u(j T)-\sum_{j=0}^{k-1} \rho^{k-1-j} h(j T) .
\end{aligned}
$$

In order to save on notation, further in the paper we will use $\mathrm{k}$ as the independent variable in place of $k T$.

Let us consider the following discrete time model of the analyzed inventory system

$$
\begin{aligned}
\mathbf{x}(k+1) & =\mathbf{A x}(k)+\mathbf{b} u(k)+\mathbf{v} h(k) \\
y(k) & =\mathbf{q}^{T} \mathbf{x}(k),
\end{aligned}
$$

where $\mathbf{x}(k)=\left[x_{1}(k), x_{2}(k), \ldots, x_{n}(k)\right]^{T}$ is the state vector, $x_{1}(k)=y(k)$ is the on-hand stock level at time instant $k$ and $x_{j}(k)=u(k-n+j-1)$ for any $j=2, \ldots, n$ represents delayed input signal $u$. Furthermore, $\mathbf{A}$ is $n \times n$ state matrix, whereas $\mathbf{b}, \mathbf{v}$, and $\mathbf{q}$ are $n \times 1$ vectors

$$
\mathbf{A}=\left[\begin{array}{ccccc}
\rho & \alpha & 0 & \cdots & 0 \\
0 & 0 & 1 & \cdots & 0 \\
\vdots & \vdots & \vdots & \ddots & \vdots \\
0 & 0 & 0 & \cdots & 1 \\
0 & 0 & 0 & \cdots & 0
\end{array}\right], \mathbf{b}=\left[\begin{array}{c}
0 \\
0 \\
\vdots \\
0 \\
1
\end{array}\right], \mathbf{v}=\left[\begin{array}{c}
-1 \\
0 \\
0 \\
\vdots \\
0
\end{array}\right], \mathbf{q}=\left[\begin{array}{c}
1 \\
0 \\
\vdots \\
0 \\
0
\end{array}\right]
$$

The system order is equal to $n=n_{p}+1=L_{p} / T+1$ and it depends on the review period and lead-time $L_{p}$.

The desired system state vector is defined as

$$
\mathbf{x}_{d}=\left[\begin{array}{c}
x_{d 1} \\
x_{d 2} \\
\vdots \\
x_{d n-1} \\
x_{d n}
\end{array}\right]=\left[\begin{array}{c}
1 \\
(1-\rho) / \alpha \\
\vdots \\
(1-\rho) / \alpha \\
(1-\rho) / \alpha
\end{array}\right] y_{r e f}
$$


where $y_{r e f}$ denotes the reference stock level. Our main objective is to stabilize the first state variable at the reference level. Therefore, when choosing the desired state vector, it is necessary to take into account that the commodities perish at the rate $1-\rho$ when kept in the warehouse as well the fact that they decay during the transportation process. Substituting the proposed desired state vector into the state equation, one can verify that in the steady state, the on-hand stock is refilled by incoming shipments at the rate equal to $y_{r e f}(1-\rho) / \alpha$.

\section{Controller design}

In this section we present the controller design procedure for the considered inventory system (5) - (6) with perishable goods and transportation losses. The procedure is based on the discrete time sliding mode approach. First, we describe the choice of the sliding hyperplane ensuring dead-beat performance. We formulate and prove the most important properties of the proposed inventory management policy. Then, we show how the system dynamical properties can be improved by applying nonlinear control based on the reaching law concept.

\subsection{Dead-beat sliding mode controller design}

For the sliding mode controller design purpose we introduce a sliding hyperplane

$$
s(k)=\mathbf{c}^{T} \mathbf{e}(k)=0
$$

where $\mathbf{c}=\left[c_{1}, c_{2}, \ldots, c_{n}\right]^{T}$ is such a vector that $\mathbf{c}^{T} \mathbf{b} \neq 0$. Parameters $c_{1}, c_{2}, \ldots, c_{n}$ will be determined further in this section. The closed-loop system error may be expressed as $\mathbf{e}(k)=\mathbf{x}_{d}-\mathbf{x}(k)$. Substituting (5) into equation $\mathbf{c}^{T} \mathbf{e}(k+1)=0$ we obtain the following control law

$$
u(k)=\left(\mathbf{c}^{T} \mathbf{b}\right)^{-1} \mathbf{c}^{T}\left[\mathbf{x}_{d}-\mathbf{A x}(k)\right] .
$$

One can easily notice from (9), that the controller properties depend on the choice of the sliding plane parameters $c_{1}, c_{2}, \ldots, c_{n}$. Using (6) we can rewrite (9) as

$$
u(k)=c_{n}^{-1}\left\{c_{1}\left[y_{\text {ref }}-\rho x_{1}(k)-\alpha x_{2}(k)\right]+\frac{(1-\rho)}{\alpha} y_{\text {ref }} \sum_{j=2}^{n} c_{j}-\sum_{j=3}^{n} c_{j-1} x_{j}(k)\right\} .
$$

In order to find such parameters of the hyperplane which will ensure that the system error is eliminated in finite (and the smallest possible) number of control steps, we analyze coefficients of the characteristic polynomial of the closed-loop system state matrix $\mathbf{A}_{c}=$ $\left[\mathbf{I}_{n}-\mathbf{b}\left(\mathbf{c}^{T} \mathbf{b}\right)^{-1} \mathbf{c}^{T}\right] \mathbf{A}$. The polynomial can be expressed as follows

$$
\operatorname{det}\left(z \mathbf{I}_{n}-\mathbf{A}_{c}\right)=z^{n}+\frac{c_{n-1}-\rho c_{n}}{c_{n}} z^{n-1}+\ldots+\frac{\alpha c_{1}-\rho c_{2}}{c_{n}} z .
$$


A discrete time system is asymptotically stable if and only if all its eigenvalues are located inside the unit circle on the $z$ plane. Additionally, for the dead-beat performance, the characteristic polynomial of the closed-loop system should have the following form

$$
\operatorname{det}\left(z \mathbf{I}_{n}-\mathbf{A}_{c}\right)=z^{n}
$$

which is satisfied when

$$
c_{n-1}=\rho c_{n}, c_{n-2}=\rho c_{n-1}, \ldots, c_{2}=\rho c_{3}, c_{1}=\frac{\rho c_{2}}{\alpha} .
$$

From (13) we immediately obtain the following vector $\mathbf{c}$ describing the parameters of the sliding plane

$$
\mathbf{c}^{T}=\left[\begin{array}{llllll}
\frac{\rho^{n-1}}{\alpha} & \rho^{n-2} & \rho^{n-3} & \ldots & \rho & 1
\end{array}\right] c_{n},
$$

which ensures that the closed-loop system has all its eigenvalues located in the origin of the $z$ plane.

Substituting (14) into (10) we obtain the control law

$$
u(k)=\frac{y_{\text {ref }}}{\alpha}-\frac{\rho^{n}}{\alpha} x_{1}(k)-\sum_{j=2}^{n} \rho^{n-j+1} x_{j}(k) .
$$

According to the state space representation of inventory system (6) the first state variable denotes the on-hand stock level $x_{1}(k)=y(k)$, and the other state variables are equal to the control signal generated at the previous $n-1$ review periods $x_{j}(k)=u(k-n+j-1)$. Therefore, taking into account that $n=n_{p}+1$, we obtain

$$
u(k)=\frac{y_{r e f}}{\alpha}-\frac{\rho^{n_{p}+1}}{\alpha} y(k)-\sum_{j=k-n_{p}}^{k-1} \rho^{k-j} u(j) .
$$

One of the fundamental issues in practical implementation of each inventory management policy is to ensure that the amount of goods shipped to the warehouse is always nonnegative and upper bounded. Therefore, now we introduce a lemma and a theorem which show that the proposed policy indeed ensures these two highly desirable properties.

First of all, one can easily notice from (16) that $u(0)=y_{\text {ref }} / \alpha$. Furthermore, for any $k \geqslant 1$ the following lemma holds.

Lemma 1 If the proposed inventory policy is applied, then for any $k \geqslant 1$

$$
u(k)=(1-\rho) \frac{y_{r e f}}{\alpha}+\frac{\rho^{n_{p}+1}}{\alpha} h(k-1) .
$$


ProOF Substituting (4) into (16), we get

$$
\begin{aligned}
u(k) & =\frac{y_{r e f}}{\alpha}-\frac{\rho^{n_{p}+1}}{\alpha}\left[\alpha \sum_{j=0}^{k-n_{p}-1} \rho^{k-n_{p}-1-j} u(j)-\sum_{j=0}^{k-1} \rho^{k-1-j} h(j)\right]-\sum_{j=k-n_{p}}^{k-1} \rho^{k-j} u(j)= \\
& =\frac{y_{r e f}}{\alpha}-\sum_{j=0}^{k-n_{p}-1} \rho^{k-j} u(j)-\sum_{j=k-n_{p}}^{k-1} \rho^{k-j} u(j)+\frac{\rho^{n_{p}}}{\alpha} \sum_{j=0}^{k-1} \rho^{k-j} h(j)= \\
& =\frac{y_{r e f}}{\alpha}-\sum_{j=0}^{k-1} \rho^{k-j} u(j)+\frac{\rho^{n_{p}}}{\alpha} \sum_{j=0}^{k-1} \rho^{k-j} h(j) .
\end{aligned}
$$

For $k=1$, it follows immediately from (18) that

$$
u(1)=\frac{y_{r e f}}{\alpha}-\rho u(0)+\frac{\rho \rho^{n_{p}}}{\alpha} h(0)=(1-\rho) \frac{y_{r e f}}{\alpha}+\frac{\rho^{n_{p}+1}}{\alpha} h(0),
$$

which shows that the lemma indeed holds for $k=1$. Now let us assume that (17) is true for all integers up to some $l>1$. Using this assumption and (18), the order quantity generated at time instant $l+1$ can be expressed as

$$
\begin{aligned}
& u(l+1)=\frac{y_{r e f}}{\alpha}-\sum_{j=0}^{l} \rho^{l+1-j} u(j)+\frac{\rho^{n_{p}}}{\alpha} \sum_{j=0}^{l} \rho^{l+1-j} h(j)= \\
& =\frac{y_{r e f}}{\alpha}+\rho \frac{y_{r e f}}{\alpha}-\rho \frac{y_{r e f}}{\alpha}-\rho \sum_{j=0}^{l-1} \rho^{l-j} u(j)-\rho u(l)+\frac{\rho^{n_{p}+1}}{\alpha} \sum_{j=0}^{l-1} \rho^{l-j} h(j)+\frac{\rho^{n_{p}+1}}{\alpha} h(l)= \\
& =\frac{y_{r e f}}{\alpha}-\rho \frac{y_{r e f}}{\alpha}+\rho\left[\frac{y_{r e f}}{\alpha}-\sum_{j=0}^{l-1} \rho^{l-j} u(j)+\frac{\rho^{n_{p}}}{\alpha} \sum_{j=0}^{l-1} \rho^{l-j} h(j)\right]-\rho u(l)+\frac{\rho^{n_{p}+1}}{\alpha} h(l)= \\
& =(1-\rho) \frac{y_{r e f}}{\alpha}+\rho u(l)-\rho u(l)+\frac{\rho^{n_{p}+1}}{\alpha} h(l)=(1-\rho) \frac{y_{r e f}}{\alpha}+\frac{\rho^{n_{p}+1}}{\alpha} h(l) .
\end{aligned}
$$

Since $l$ is an arbitrary positive integer, it follows from the principle of mathematical induction that (17) is true for any integer $k \geqslant 1$. This ends the proof of the lemma.

Theorem 1 If the proposed warehouse management policy is applied, then for any $k \geqslant 0$ the control signal satisfies the following inequalities

$$
(1-\rho) \frac{y_{r e f}}{\alpha} \leqslant u(k) \leqslant \max \left[\frac{y_{r e f}}{\alpha},(1-\rho) \frac{y_{r e f}}{\alpha}+\frac{\rho^{n_{p}+1}}{\alpha} d_{\max }\right] .
$$

PROOF It follows directly from (16) that $u(0)=y_{r e f} / \alpha$, and this implies that the theorem is satisfied for $k=0$. Moreover, since the demand is always bounded as stated by inequalities (1), then for any $k>0$, from Lemma 1 , we obtain

$$
(1-\rho) \frac{y_{r e f}}{\alpha} \leqslant u(k) \leqslant(1-\rho) \frac{y_{r e f}}{\alpha}+\frac{\rho^{n_{p}+1}}{\alpha} d_{\max },
$$


which concludes the proof of Theorem 1.

The next theorem states another important property of the proposed policy, namely it shows that the inventory level never exceeds its reference value. This proposition shows that if the warehouse capacity is selected at least equal to $y_{\text {ref }}$, then enough storage space at the distribution centre for all incoming shipments will always be provided.

Theorem 2 If the proposed inventory management policy is applied, then for any $k \geqslant 0$ the stock level is always upper bounded by $y_{\text {ref }}$, i.e.

$$
y(k) \leqslant y_{r e f} .
$$

PROOF Due to initial conditions and lead-time delay, the considered warehouse is empty for any $k \leqslant n_{p}=n-1$. Hence, we need to show that inequality (23) holds for any $k \geqslant n$.

Let us assume that for some integer $l \geqslant n, y(l) \leqslant y_{\text {ref }}$. Then, we will demonstrate that this inequality is also satisfied for $l+1$. The stock level at the time instant $l+1$, based on the inventory balance equation, can be expressed as

$$
y(l+1)=\rho y(l)+\alpha u\left(l-n_{p}\right)-h(l) .
$$

Substituting (4) and (18) into (24), we obtain

$$
\begin{aligned}
& y(l+1)=\rho y(l)+\alpha \frac{y_{\text {ref }}}{\alpha}-\alpha \rho \sum_{j=0}^{l-n_{p}-1} \rho^{l-n_{p}-1-j} u(j)+\rho^{n_{p}+1} \sum_{j=0}^{l-n_{p}-1} \rho^{l-n_{p}-1-j} h(j)-h(l)= \\
& \quad=\rho y(l)+y_{\text {ref }}-\rho y(l)-\sum_{j=l-n_{p}}^{l-1} \rho^{l-1-j} h(j)-h(l)= \\
& \quad=y_{\text {ref }}-\sum_{j=l-n_{p}}^{l} \rho^{l-j} h(j) .
\end{aligned}
$$

Since $h(k)$ is always nonnegative, then $y(l+1) \leqslant y_{\text {ref }}$. Using the principle of the mathematical induction we conclude that the theorem is satisfied for any $k \geqslant 0$.

Now we will formulate and prove the last theorem, which shows how to select the reference stock level, so that full demand satisfaction is guaranteed. In other words, this theorem demonstrates how big warehouse capacity is needed, to ensure that all sales are realized from the readily available resources.

Theorem 3 If the proposed inventory policy is applied, and the target stock level satisfies the following inequality

$$
y_{\text {ref }}>d_{\max } \sum_{j=0}^{n_{p}} \rho^{j},
$$

then for any $k \geqslant n$ the stock level is strictly positive. 
ProOF Assumption (1) implies that the realized demand is always upper bounded. Hence, taking into account (25) and (26), for any $k \geqslant n$, we obtain

$$
\begin{aligned}
y(k) & =y_{\text {ref }}-\sum_{j=k-1-n_{p}}^{k-1} \rho^{k-1-j} h(j) \geqslant \\
& \geqslant y_{\text {ref }}-d_{\max } \sum_{j=k-1-n_{p}}^{k-1} \rho^{k-1-j}=y_{r e f}-d_{\max } \sum_{j=0}^{n_{p}} \rho^{j}>0 .
\end{aligned}
$$

This concludes the proof.

Notice that the reference stock level required to ensure full demand satisfaction does not depend on the transport decay factor $\alpha$. Hence, the decrease of this factor does not influence the size of the warehouse. However, as it can be seen from (16), the order quantity generated by the proposed policy rises with the decrease of coefficient $\alpha$.

\subsection{Reaching law based SM controller design}

Closer analysis of the proposed dead-beat controller performance reveals that it may generate excessive orders in the initial phase of the regulation process. Therefore, in this section we propose a modified controller which maintains favourable dynamic properties of the dead-beat scheme, but also ensures that the amount of ordered goods is always limited, as required by suppliers. For that purpose, we apply the concept of the reaching law in the form presented by Golo and Milosavljević [11]. This reaching law lets us specify how the system representative point approaches the sliding hyperplane and consequently makes it possible to extend the reaching phase over several time instants, instead of requiring the system representative point to reach the hyperplane in one step. Further in this paper, we will prove that proper selection of the reaching law ensures satisfaction of the following input constraint

$$
0 \leqslant u(k) \leqslant u_{\max },
$$

where $u_{\max }>(1-\rho) y_{\text {ref }} / \alpha+\left(\rho^{n} / \alpha\right) d_{\max }$, and at the same time enables preserving the advantages offered by the dead-beat scheme.

The reaching law described in [11] can be expressed in the following manner

$$
s(k+1)-s(k)=-\Phi[s(k)],
$$

where

$$
\Phi[s(k)]=\min \left\{|s(k)|, \delta_{1}|s(k)|+\delta_{2}\right\} \operatorname{sgn}[s(k)],
$$

$0 \leqslant \delta_{1}<1$, and $\delta_{2}>0$. The $\operatorname{sgn}(x)$ function in (30) is defined as follows

$$
\operatorname{sgn}(x)=\left\{\begin{array}{rll}
-1 & \text { for } & x \leqslant 0 \\
1 & \text { for } & x>0
\end{array}\right.
$$


If this reaching law is applied, then the system representative point is guaranteed to reach the hyperplane $s(k)=\mathbf{c}^{T} \mathbf{e}(k)=0$ monotonically in a finite number of steps in a way determined by the choice of coefficients $\delta_{1}$ and $\delta_{2}$. In our further analysis, we express the reaching law determined by (29) and (30) in the alternative way

$$
s_{1}(k)=\mathbf{c}^{T} \mathbf{e}(k)+f(k)=0,
$$

where strictly monotonic function $f(\cdot)$ has the following form

$$
\begin{cases}f(k+1)=\left(1-\delta_{1}\right) f(k)-\delta_{2} \operatorname{sgn}[f(k)] & \text { for } k<k_{0}, k_{0} \in C_{+}, \\ f(k+1)=0 & \text { for } k \geqslant k_{0}\end{cases}
$$

and $f(0)=-\mathbf{c}^{T} \mathbf{e}(0)=-c_{n} y_{r e f} / \alpha$. Since $f(\cdot)$ is strictly monotonic this also implies that for any $0 \leqslant k<k_{0}$ function $f(\cdot)$ and coefficient $c_{n}$ have opposite signs. Notice that function $f(\cdot)$ reflects the distance which needs to be covered by the system representative point before it reaches the desired sliding plane $\mathbf{c}^{T} \mathbf{e}(k)=0$. Hence, we need to choose parameters $\delta_{1}$ and $\delta_{2}$ so that input constraint (28) is satisfied. Substituting equations (5) into $\mathbf{c}^{T} \mathbf{e}(k+1)+f(k+1)=0$ we obtain

$$
u(k)=\left(\mathbf{c}^{T} \mathbf{b}\right)^{-1}\left\{\mathbf{c}^{T}\left[\mathbf{x}_{\mathbf{d}}-\mathbf{A} \mathbf{x}(k)\right]+f(k+1)\right\} .
$$

Then applying (14) we get

$$
u(k)=\frac{y_{r e f}}{\alpha}-\frac{\rho^{n_{p}+1}}{\alpha} y(k)-\sum_{j=k-n_{p}}^{k-1} \rho^{k-j} u(j)+\frac{f(k+1)}{c_{n}} .
$$

Firstly, we state the relation between the control signal established according to (35) and the realized demand $h(\cdot)$. Next, we describe selection of the reaching law parameters.

At the first time instant we get

$$
u(0)=\frac{y_{r e f}}{\alpha}+\frac{f(1)}{c_{n}} .
$$

Furthermore, for any $k \geqslant 1$, the control signal satisfies the following lemma.

Lemma 2 If policy (35) is applied to the considered supply chain, then for any $k \geqslant 1$

$$
u(k)=(1-\rho) \frac{y_{r e f}}{\alpha}+\frac{\rho^{n_{p}+1}}{\alpha} h(k-1)+\frac{f(k+1)-\rho f(k)}{c_{n}} .
$$

PrOOF Substituting (4) into (35), we get

$$
u(k)=\frac{y_{r e f}}{\alpha}-\frac{\rho^{n_{p}+1}}{\alpha}\left[\alpha \sum_{j=0}^{k-n_{p}-1} \rho^{k-n_{p}-1-j} u(j)-\sum_{j=0}^{k-1} \rho^{k-1-j} h(j)\right]+
$$




$$
\begin{aligned}
& -\sum_{j=k-n_{p}}^{k-1} \rho^{k-j} u(j)+\frac{f(k+1)}{c_{n}}= \\
& =\frac{y_{r e f}}{\alpha}-\sum_{j=0}^{k-1} \rho^{k-j} u(j)+\frac{\rho^{n_{p}}}{\alpha} \sum_{j=0}^{k-1} \rho^{k-j} h(j)+\frac{f(k+1)}{c_{n}} .
\end{aligned}
$$

For $k=1$, we obtain

$$
\begin{aligned}
u(1) & =\frac{y_{r e f}}{\alpha}-\rho u(0)+\frac{\rho \rho^{n_{p}}}{\alpha} h(0)+\frac{f(2)}{c_{n}}= \\
& =(1-\rho) \frac{y_{r e f}}{\alpha}+\frac{\rho^{n_{p}+1}}{\alpha} h(0)+\frac{f(2)-\rho f(1)}{c_{n}}
\end{aligned}
$$

which shows that the lemma is indeed satisfied for $k=1$. Let us assume that (37) is true for all integers up to some $l>1$. Using this assumption, the order quantity generated in period $l+1$ can be expressed in the following way

$$
\begin{aligned}
u(l+1) & =\frac{y_{r e f}}{\alpha}-\sum_{j=0}^{l} \rho^{l+1-j} u(j)+\frac{\rho^{n_{p}}}{\alpha} \sum_{j=0}^{l} \rho^{l+1-j} h(j)+\frac{f(l+2)}{c_{n}}= \\
& =\frac{y_{r e f}}{\alpha}+\rho \frac{y_{r e f}}{\alpha}-\rho \frac{y_{r e f}}{\alpha}-\rho \sum_{j=0}^{l-1} \rho^{l-j} u(j)-\rho u(l)+ \\
& +\frac{\rho^{n_{p}+1}}{\alpha} \sum_{j=0}^{l-1} \rho^{l-j} h(j)+\frac{\rho^{n_{p}+1}}{\alpha} h(l)+\frac{f(l+2)}{c_{n}}= \\
& =\frac{y_{r e f}}{\alpha}-\rho \frac{y_{r e f}}{\alpha}+\rho u(l)-\rho u(l)+\frac{\rho^{n_{p}+1}}{\alpha} h(l)-\frac{\rho f(l+1)}{c_{n}}+\frac{f(l+2)}{c_{n}} \\
& =(1-\rho) \frac{y_{r e f}}{\alpha}+\frac{\rho^{n_{p}+1}}{\alpha} h(l)+\frac{f(l+2)-\rho f(l+1)}{c_{n}} .
\end{aligned}
$$

Since $l$ is an arbitrary integer greater than zero, it follows immediately from the principle of mathematical induction that (37) is true for any integer $k \geqslant 1$. This concludes the proof of the lemma.

The comparison of relations (33) and (37) leads to the conclusion that a suitable choice for $\delta_{1}$ is the decay factor $\sigma=1-\rho$. Now we need to select $\delta_{2}$ so that $u(k)$ is always smaller than or equal to $u_{\max }$. Substituting (33) into (37) and putting $\delta_{1}=1-\rho$ we get

$$
u(k)= \begin{cases}(1-\rho) \frac{y_{r e f}}{\alpha}+\frac{\rho^{n_{p}+1}}{\alpha} h(k-1)-\frac{\delta_{2} \operatorname{sgn}[f(k)]}{c_{n}} & \text { for } k<k_{0}, \\ (1-\rho) \frac{y_{r e f}}{\alpha}+\frac{\rho^{n_{p}+1}}{\alpha} h(k-1) & \text { for } k \geqslant k_{0} .\end{cases}
$$

It follows immediately from assumption (1) that the control signal is nonnegative and upper bounded by $(1-\rho) y_{\text {ref }} / \alpha+\left(\rho^{n} / \alpha\right) d_{\max }<u_{\max }$ for any $k \geqslant k_{0}$. The consequence 
of lead-time delay is that no request placed at the distribution centre can be realized until the first order arrives after $n_{p}$ time instants. Furthermore, according to our assumptions $h\left(k<n_{p}\right)=0$.

In order to ensure that condition (28) is true for all $k<k_{0}$ we conclude that parameter $\delta_{2}$ should satisfy one of the following constraints, depending on time instant,

$$
u(k)= \begin{cases}\delta_{2} \leqslant\left|c_{n}\right|\left[u_{\max }-(1-\rho) \frac{y_{\text {ref }}}{\alpha}\right] & \text { for } 0 \leqslant k \leqslant n_{p} \\ \delta_{2} \leqslant\left|c_{n}\right|\left[u_{\max }-(1-\rho) \frac{y_{\text {ref }}}{\alpha}+\frac{\rho^{n_{p}+1}}{\alpha} d_{\max }\right] & \text { for } k>n_{p} .\end{cases}
$$

This concludes the design of the reaching law. The obtained controller generates the quantity of orders on the basis of equation (35) with function $f(\cdot)$ defined by (33). Notice that parameters of function $f(\cdot)$ are selected as $\delta_{1}=1-\rho$ and $\delta_{2}$ as the biggest value satisfying inequalities (42). Now the most important properties of the nonlinear controller will be formulated as two theorems.

Theorem 4 If the proposed inventory management policy (35) is applied, then the on-hand stock never exceeds $y_{\text {ref }}$.

PROOF The warehouse at the distribution centre is initially empty and remains empty for any $k<n_{p}$. Hence, we need to show that the proposition is also satisfied for all $k>n_{p}$. Using Lemma 2, the amount of goods in the warehouse given by (4) can be expressed as

$$
\begin{aligned}
& y(k)=\alpha \rho^{k-n_{p}-1} u(0)+\alpha \sum_{j=1}^{k-n_{p}-1} \rho^{k-n_{p}-1-j} u(j)-\sum_{j=0}^{k-1} \rho^{k-1-j} h(j)= \\
& =\rho^{k-n_{p}-1} y_{r e f}+\alpha \rho^{k-n_{p}-1} \frac{f(1)}{c_{n}}+\alpha \sum_{j=1}^{k-n_{p}-1} \rho^{k-n_{p}-1-j}\left[(1-\rho) \frac{y_{r e f}}{\alpha}+\frac{\rho^{n_{p}+1}}{\alpha} h(j-1)\right]+ \\
& +\alpha \sum_{j=1}^{k-n_{p}-1} \rho^{k-n_{p}-1-j} \frac{f(j+1)-\rho f(j)}{c_{n}}-\sum_{j=0}^{k-1} \rho^{k-1-j} h(j) .
\end{aligned}
$$

Furthermore,

$$
\begin{aligned}
y(k) & =\rho^{k-n_{p}-1} y_{\text {ref }}+(1-\rho) y_{\text {ref }} \sum_{j=1}^{k-n_{p}-1} \rho^{k-n_{p}-1-j}+ \\
& +\sum_{j=1}^{k-n_{p}-1} \rho^{k-j} h(j-1)-\sum_{j=0}^{k-1} \rho^{k-1-j} h(j)+\frac{\alpha f\left(k-n_{p}\right)}{c_{n}}= \\
& =\rho^{k-n_{p}-1} y_{r e f}+\left(1-\rho^{k-n_{p}-1}\right) y_{r e f}-\sum_{j=k-n_{p}-1}^{k-1} \rho^{k-1-j} h(j)+\frac{\alpha f\left(k-n_{p}\right)}{c_{n}}=
\end{aligned}
$$




$$
=y_{r e f}-\sum_{j=k-n_{p}-1}^{k-1} \rho^{k-1-j} h(j)+\frac{\alpha f\left(k-n_{p}\right)}{c_{n}} .
$$

Since the satisfied demand $h(\cdot)$ is always nonnegative, and $f(k)$ and $c_{n}$ have opposite signs for all $k>0, y(k)$ is always upper-bounded by $y_{\text {ref }}$. This completes the proof of Theorem 4.

Theorem 5 If policy (35) is applied to the considered supply chain and the reference stock level satisfies $y_{\text {ref }}>d_{\max } \sum_{j=0}^{n_{p}} \rho^{j}$, then for any $k \geqslant n+k_{0}$ the amount of goods in the warehouse is strictly positive and demand is entirely satisfied from the readily available resources.

PROOF It follows from relation (33) that for $k>k_{0}$ function $f(k)=0$. As a consequence for $k \geqslant k_{0}$, the nonlinear controller (35) becomes equivalent to linear control law (16), whose action influences the stock level for $k \geqslant n+k_{0}$. Since both controllers incorporate the order history exactly in the same way, then we conclude that the proposition is valid as a direct consequence of the Theorem 3. This completes the proof of Theorem 5.

\section{Numerical example}

In order to verify effectiveness of the proposed supply policies we perform a series of simulation tests. Parameters of the system are chosen as follows: review period $T=1$ day, lead-time delay $L_{p}=n_{p} T=6$ days, transport decay factor $\alpha=0.92$, inventory deterioration rate $\sigma=0.05$ (which results in $\rho=1-0.05=0.95$ ), and the maximum daily demand at the distribution centre $d_{\max }=70$ items. The imposed demand at the distribution centre is illustrated in Fig. 3. This demand pattern is chosen to verify performance of both controllers in the presence of abrupt changes of the demand. In order to guarantee maximum service level, i.e. full demand satisfaction, according to Theorem 3 , the reference stock level for supply policies (16) and (35) should be greater than 423 items. Thus, we select $y_{\text {ref }}$ equal to 430 items. For the reaching law based supply strategy, it is assumed that supplier cannot provide more goods in a single review period than $u_{\max }$ equal to 100 items. The parameters of the reaching law based controller are chosen in the following way: $\delta_{1}=1-\rho=0.05, \delta_{2}=77.01$ for $0 \leqslant k \leqslant 6$ and $\delta_{2}=23.88$ for $k>6$ (both calculated according to inequalities (42)).

The orders generated by the proposed policies are shown in Fig. 4 and the on-hand stock in Fig. 5. It can be easily seen from Fig. 4, that after the initial phase the control signal generated by policy (16) is identical to the one generated by policy (35), i.e. after $k_{0}$ time instants both policies become equivalent. Moreover, it is worth to point out that during the initial phase, the control signal generated by policy (35) remains constant and equal to $u_{\max }$. This is a direct consequence of the proper choice of the reaching 


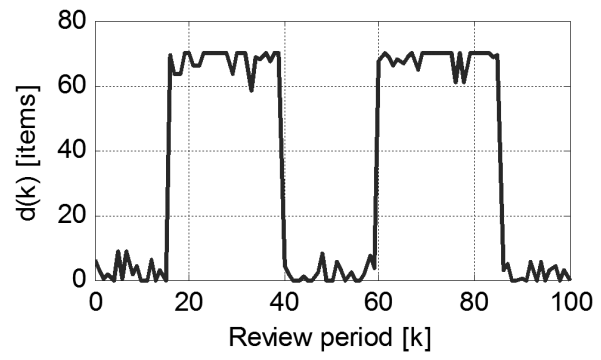

Figure 3. The imposed demand at the distribution center.

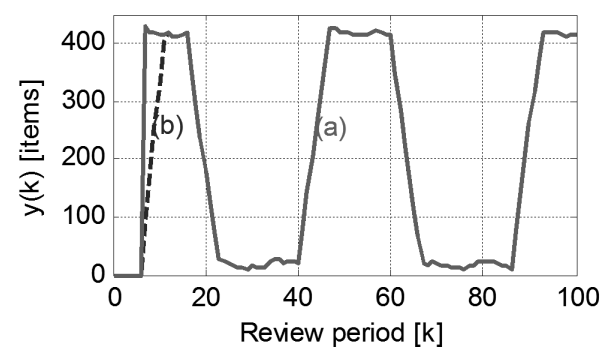

Figure 5. On-hand stock level: a) policy (16), b) policy (35).

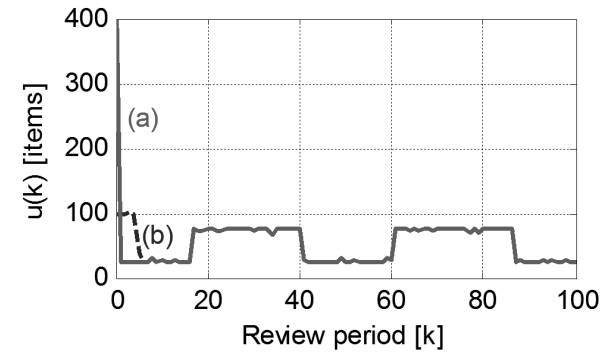

Figure 4. Order quantities: a) policy $(16)$, b) policy (35).

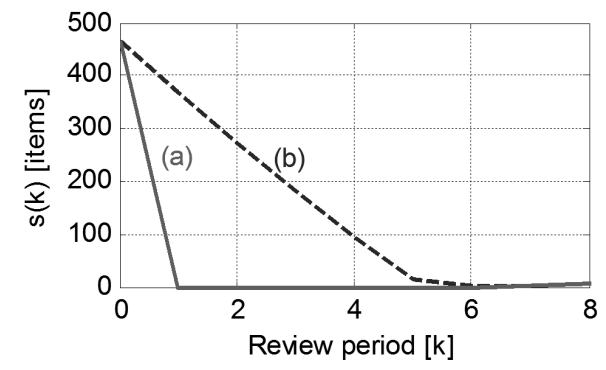

Figure 6. Sliding variable $s(k)$ : a) policy $(16)$, b) policy (35).

law parameters, which results in the increase of the sum in (35) being compensated by the same change of function $f(\cdot)$. Furthermore, it can be noticed from Fig. 5 that in both cases the on-hand stock level does not exceed the warehouse capacity, and after the initial phase it never drops to zero. Finally, Fig. 6 shows that the representative point of the system controlled according to (16) reaches the sliding hyperplane in one step, precisely as assumed in the design procedure, whereas controller (35) reduces the magnitude of the sliding variable during the first 6 time instants.

\section{Conclusions}

In this paper a new discrete time, chattering-free sliding mode controller for supply chains with deteriorating stock and commodity loses in the order procurement process is designed. The controller employs a sliding hyperplane which is designed in such a way that the closed loop system exhibits the dead-beat dynamic performance. In other words, the controller reacts fast to the unpredictable variations of demand and ensures stable system operation for arbitrary positive lead-time. Moreover, the proposed controller ensures $100 \%$ demand satisfaction and guarantees that the stock level never exceeds the warehouse capacity (in this way it eliminates the need of hiring, usually very costly, ex- 
tra storage space out of the company premises). Since the proposed dead-beat sliding mode controller may generate unacceptably big initial value of the control signal, and therefore require excessive shipments at the beginning of the control process, we also propose an alternative controller based on the reaching law concept. The controller, on one hand preserves good system dynamics of the original strategy and on the other ensures that input signal constraint is satisfied. When the modified controller is applied, the on-hand stock level is always upper bounded and never drops to zero after the initial phase of the control process. This implies that the warehouse capacity is not exceeded and that the entire imposed demand is satisfied (thus necessity of backorders is eliminated). These favorable properties have been formulated as theorems, formally proved and verified in a simulation example. Both control strategies proposed in this paper are computationally efficient and simple in software implementation.

\section{References}

[1] E. Aggelogiannaki, P. Doganis and H. Sarimveis: An adaptive model predictive control configuration for production-inventory systems. Int. J. of Production Economics, 114(1), (2008), 165-178.

[2] B. BAndyopadhyay and S. JanaRdhanan: Discrete-time sliding mode control: a multirate output feedback approach LNCIS. 323, Springer-Verlag, Berlin Heidelberg, 2006.

[3] A. Bartoszewicz: Discrete-time quasi-sliding-mode control strategies. IEEE Trans. on Industrial Electronics, 45(4), (1998), 633-637.

[4] A. Bartoszewicz: Remarks on 'Discrete-time variable structure control systems'. IEEE Trans. on Industrial Electronics, 43(1), (1996), 235-238.

[5] M. Boccadoro, F. Martinelli and P. Valigi: Supply chain management by $H_{\infty}$ control. IEEE Trans. on Automation Science and Engineering, 5(4), (2008), 703-707.

[6] E-K. Boukas, P. ShI and R.K. Agarwal: An application of robust technique to manufacturing systems with uncertain processing time. Optimal Control Applications and Methods, 21(6), (2000), 257-268.

[7] K. FuRUTA: Sliding mode control of a discrete system. Systems and Control Letters, 14 (1990), 145-152.

[8] G. GAALMAN: Bullwhip reduction for ARMA demand: the proportional order-upto policy versus the full-state-feedback policy. Automatica: 42(8), (2006), 12831290. 
[9] G. GAALMAN and S.M. DiSNEY: State space investigation of the bullwhip problem with ARMA(1,1) demand processes. Int. J. of Production Economics, 104(2), (2006), 327-339.

[10] W. GaO, Y. WANG and A. HomaIFA: Discrete-time variable structure control system. IEEE Trans. on Industrial Electronics, 42 (1995), 117-122.

[11] G. Golo and C. Milos avljević: Robust discrete-time chattering free sliding mode control. Systems and Control Letters, 41 (2000), 19-28.

[12] K. Hoberg, J. R. Bradley and U.W. Thonemann: Analyzing the effect of the inventory policy on order and inventory variability with linear control theory. European J. of Operational Research, 176(3), (2007), 1620-1642.

[13] P. IgNaCiuk and A. BARToszewicz: Dead-time compensation in continuousreview perishable inventory systems with a remote supply source. Archives of Control Sciences, 21(1) (2011), 5-28.

[14] P. IGNACIUK and A. BARTOSZEwiCZ: Linear-quadratic optimal control of periodic-review perishable inventory systems. IEEE Trans. on Control Systems Technology, 20(3), (2012), 1400-1407.

[15] P. Ignaciuk and A. Bartoszewicz: Linear-quadratic optimal control strategy for periodic-review inventory systems. Automatica, 46(12), (2010), 1982-1993.

[16] P. IgNACIUK and A. BARToszewicz: LQ optimal and reaching law based design of sliding mode supply policy for inventory management systems. Archives of Control Sciences, 19(3), (2009), 261-277.

[17] P. IGNACIUK and A. BARTOSZEWICZ: LQ optimal sliding mode supply policy for periodic inventory systems. IEEE Trans. on Automatic Control, 55(1), (2010), 269-274.

[18] P. IgnaCiUK and A. BARToszewiCZ: LQ optimal sliding mode supply policy for periodic-review perishable inventory systems. J. of the Franklin Institute, 349(4), (2012), 1561-1582.

[19] P. Ignaciuk and A. BARToszewicz: Dead-beat and reaching-law-based slidingmode control of perishable inventory systems. Bulletin of the Polish Academy of Sciences - Technical Sciences, 59(1), (2011), 39-49.

[20] I. Karaesmen, A. Scheller-Wolf and B. Deniz: Managing perishable and aging inventories: review and future research directions. In: Kempf K., Keskinocak P., and Uzsoy R. (Eds.) Handbook of production planning. Dordrecht: Kluwer, 2008. 
[21] X. Li and T.E. MARLIN: Robust supply chain performance via Model Predictive Control. Computers and Chemical Engineering, 33(12), (2009), 2134-2143.

[22] C. Milosavljević, B. Peruničić-Draženović, B. Veselić and D. Mitić: Sampled data quasi-sliding mode control strategies. IEEE Int. Conf. on Industrial Technology, Mumbai, (2006), 2640-2645.

[23] H. Sarimveis, P. Patrinos, C.D. Tarantilis and C.T. Kiranoudis: Dynamic modeling and control of supply chain systems: a review. Computers and Operations Research, 35(11), (2008), 3530-3561.

[24] H.A. SIMON: On the application of servomechanism theory in the study of production control. Econometrica, 20(2), (1952), 247-268.

[25] V. UtKin and S.V. DRAKunOw: On discrete-time sliding mode control. IFAC Conf. on Nonlinear Control, Capri, Italy, (1989), 484-489.

[26] H.J. VASSIAN: Application of discrete variable servo theory to inventory control. Arthur D. Little, Inc., Cambridge Massachusetts, 1954. 